\title{
Investigation d'un cas de récidive de méningite, W135 et Y à Yirimadio, Bamako, Juin 2019
}

Investigation of a case of recurrent meningitis, W135 and Y in Yirimadio, Bamako, June 2019

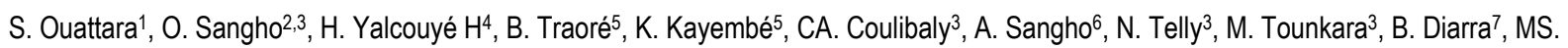
Konaké $^{7}$, HM. NDiaye ${ }^{7}, \mathrm{OB}$. Togola $^{8}$

1. Direction Régionale de la Santé de Bamako, Mali

2. DER des Sciences Biologiques et Médicales, FAPH/USTTB, Mali

3. Département d'Enseignement et de Recherche en Santé Publique, FMOS/USTTB, Mali

4. Direction Nationale des Services Vétérinaires, Bamako, Mali

5. Réseau Africain des Epidémiologiste de Terrain, AFENETMali, DGSHP, Bamako, Mali

6. Direction de la Pharmacie et du Médicament (DPM), Bamako, Mali

7. Centre de Santé de Référence de la Commune VI, Bamako, Mali

8. Direction Régionale de la Santé et de l'Hygiène Publique, Ségou

9. Centre de Santé de Référence de Tominian, Ségou, Mali

Auteur correspondant : Dr Seydou Ouattara, Direction Régionale de la Santé de Bamako, Mali, 782640 46, drouattaraseydou@gmail.com

\section{Résumé :}

La méningite est une inflammation des méninges : les enveloppes de la moelle épinière et du cerveau dans lesquelles circule le liquide céphalorachidien. Bien que les épidémies dans la ceinture méningitique soient dues à Neisseria meningitidis du sérogroupe $\mathrm{A}$, la survenue en 2000 d'une épidémie au niveau mondial a permis de mettre en cause la responsabilité de $N$. meningitidis du sérogroupe W135, et en 2016 la survenue d'une épidémie de méningite causée par $\mathrm{N}$. meningitidis du sérogroupe $C$ au Mali. Un cas de méningite W135/y a été détecté par le service d'infectiologie du CHU Point G le 18 Juin 2019. Par la suite nous avons investiguer le cas de méningite $W 135 / Y$ dans l'aire de santé de Yirimadio en commune VI dans le District de Bamako en Juin 2019. Notre objectif était décrire le cas, déterminer le potentiel de diffusion de la maladie, proposer des mesures de contrôle. II s'agissait d'un patient de 42 ans avec méningite $W 135$ et $Y$ ayant bénéficié d'une meilleure prise en charge au $\mathrm{CHU}$ contrairement au cabinet privé. La guérison a intervenu sans séquelles. Nous avons effectué un briefing du personnel du cabinet par rapport à son implication dans la surveillance épidémiologique.

Mots clés : investigation, méningite, récidive, Yirimadio, Bamako

\footnotetext{
Abstract:

Meningitis is an inflammation of the meninges: the envelopes of the spinal cord and brain in which the cerebrospinal fluid circulates. Although epidemics in the meningitis belt are due to Neisseria Meningitidis serogroup A, the occurrence in 2000 of a global epidemic has implicated $N$. meningitidis responsibility of serogroup
}

W135, and in 2016 the occurrence an epidemic of meningitis caused by $N$. meningitidis serogroup $C$ in Mali. A case of meningitis W135 / Y was detected by the Infectious Disease Unit of the teaching hospital Point G on June 18, 2019. Then we investigated the case of meningitis $\mathrm{W} 135 / \mathrm{Y}$ in the health area of Yirimadio in commune VI in the District of Bamako in June 2019. Our objective was to describe the case, to determine the diffusion potential of the disease, to propose control measures. It was a 42-year-old patient with meningitis $W 135$ and $Y$ who had better care at the hospital than the private practice. Healing took place without sequelae. We briefed the firm's staff on their involvement in epidemiological surveillance.

Keywords: investigation, meningitis, recurrent, Yirimadio, Bamako

\section{Introduction}

La méningite est une inflammation des méninges : les enveloppes de la moelle épinière et du cerveau dans lesquelles circule le liquide céphalorachidien $(1,2)$. Dans la plupart des cas, la méningite est due à un virus (méningite virale). La méningite peut aussi être due à une bactérie (méningite bactérienne). La méningite bactérienne est habituellement beaucoup plus grave que la méningite virale et nécessite des soins en urgence extrême (1). Les sérogroupes $W 135$ et $Y$ font parties des 12 connus (1).

Bien que les épidémies dans la ceinture méningitique soient dues à $N$. meningitidis du sérogroupe A (3), la survenue en 2000 d'une épidémie au niveau mondial a permis de mettre en cause la responsabilité de $N$. meningitidis du sérogroupe W135, et en 2016 la survenue d'une épidémie de méningite causée par $N$. meningitidis du sérogroupe $C$ au Mali (4). Une surveillance épidémiologique active avec renforcement des capacités du laboratoire permet une détection précoce des épidémies, une identification du sérogroupe en cause, un choix adéquat de l'antibiotique et une sélection appropriée du vaccin afin de protéger la population (4).

Avant 2010, le sérogroupe A était responsable de la grande majorité des épidémies survenues dans la ceinture de la méningite. Depuis 2010, l'introduction progressive d'un vaccin conjugué contre le méningocoque $A$ (MenAfriVac) en Afrique a entraîné une baisse spectaculaire du nombre de cas de méningite à N.m. A et l'élimination des épidémies dues à N.m. A. Parallèlement, la proportion relative de cas dus à d'autres sérogroupes ( $\mathrm{W}, \mathrm{X}$ et $\mathrm{C}$ ) et à Streptococcus 
pneumoniae (Spn) a augmenté. La maladie se transmet par voie aérienne d'une personne à l'autre par de grosses gouttelettes de sécrétions rhinopharyngées d'individus infectés. La période d'incubation dure de 2 à 10 jours. Les taux d'attaque les plus élevés sont observés chez les enfants de moins de 15 ans. Les taux de létalité sont généralement compris entre 8 et $15 \%$ chez les malades traités, et $>70 \%$ en l'absence de traitement. De nombreux survivants garderont des séquelles à long terme, notamment des retards mentaux, la perte de l'ouïe et de l'usage des membres. Les symptômes d'une méningite : la fièvre, la raideur au cou, le mal de tête (difficile à détecter chez un tout petit) : il pleure beaucoup, les vomissements, la somnolence, les convulsions, les taches rouges ou bleues sur la peau, la constipation.

La notification du cas suspect de méningite W135 a été faite par le chef de service des maladies infectieuses du Centre Hospito-Universitaire ( $\mathrm{CHU})$ du Point $\mathrm{G}$ à la Direction Générale de la Santé et l'Hygiène Publique (DGSHP) qui a saisi la Direction Régionale de Santé de Bamako pour informer le CSRéf de la commune VI d'où le malade est originaire. Le malade était domicilié à Yirimadio. Ce qui motiva l'information du Directeur Technique du Centre (DTC) de ce Centre de Santé Communautaire (CSCom). Une équipe composée des représentants de ces différentes structures auxquelles se sont ajoutés les deux participants de la cohorte intermédiaire du diplôme universitaire en épidémiologie de terrain (cohorte intermédiaire), s'est rendue au (Centre Hospitalier Universitaire) CHU-Point G et à Yirimadio pour investiguer ce cas de méningite. L'objectif était de décrire le cas suspect de méningite $W 135$ au Point $\mathrm{G}$ et à Yirimadio en juin 2019.

\section{Méthodologie : \\ Type d'étude : il s'agissait d'une étude descriptive.}

Période et lieu d'étude : l'étude s'est déroulée en juin 2019 au CHU du Point G, au CSRéf Commune VI, au CSCom de Yirimadio et au quartier de Yirimadio.

Collecte des données: Nous avons réalisé une investigation épidémiologique avec description de cas. L'équipe d'investigation était composée de cadres de la Direction Générale de la Santé et de l'Hygiène Publique (DGSHP) à préciser, du Responsable surveillance épidémiologique Direction Régionale de la Santé de Bamako (DRS), du Médecin d'appui Programme Elargi de Vaccination et Surveillance Epidémiologique (PEV/SE) du Centre de Santé de Référence de la Commune VI (CSRéf CVI), du Directeur Technique du Centre de Santé Communautaire de Yirimadio (DTC ASACOYIR), de la Direction Nationale des Services Vétérinaires (DNSV) et d'un chauffeur.

Nous avons rendu visite au malade dans le service d'infectiologie du Point-G le 06/06/2019. Nous avons interrogé le malade, ses accompagnants ainsi que le personnel de santé chargé de ses soins. Le chef de service n'était pas disponible pour l'entretien. Le
07/06/19 nous avons pu avoir accès à son dossier que nous avons exploité pour y collecter les informations sur le traitement, les examens complémentaires, les résultats et l'évolution.

Nous avons visité le CSRéf et échangé avec le médecin chef qui nous a fourni un véhicule. Nous avons successivement visité le CSCom, le Cabinet médical B. où le malade avait consulté avant d'être référé au point $G$ et le domicile du malade à Yirimadio. Nous avons eu des entretiens avec le DTC, le médecin référent (par téléphone) et les membres de la famille. Au niveau du domicile, nous avons examiné la carte de vaccination des enfants cas suspects. L'ASC a été mise à contribution (par téléphone) par le DTC, pour le suivi à domicile du patient et des membres de sa famille, la détection d'éventuel cas suspect. Nous avons recherché d'autres cas similaires dans le quartier sans succès.

Définition de cas confirmé : Tout cas présumé chez qui le $N$. meningitidis a été mis en évidence par culture ou par Polymerase Chain Reaction (PCR) au laboratoire, vivant à Yirimadio du 01 Juin 2019 au 30 Juin 2019.

Définition cas suspect : Toute personne présentant une forte fièvre d'apparition brutale (température rectale $>38,5^{\circ} \mathrm{C}$ ou axillaire $>38,0^{\circ} \mathrm{C}$ ) et l'un des signes suivants : raideur de la nuque, altérations de la conscience ou autres signes méningés, vivant à Yirimadio du 01 Juin 2019 au 30 Juin 2019.

Nous avons utilisé un bloc note pour collecter les informations.

Traitement et analyse des données: Les variables collectées étaient entre autres: l'âge, le sexe, la profession, la résidence, les dates de début, consultation, prélèvement et de notification, les circonstances de découverte, les antécédents médicaux, l'évolution. Nous avons procédé à une description littéraire simple des données.

Les considérations éthiques et déontologiques ont été respectées. Les noms du malade ne sont pas ressortis, aucune information dans ce rapport ne permet de remonter jusqu'au malade. Aucune question gênante ne lui a été posée. L'objet des différentes interviews était de pouvoir comprendre ce cas afin de pouvoir éviter d'autres cas et arrêter la réinfection.

\section{Résultats :}

Description du cas :

II s'agissait de BT, un homme âgé de 42 ans, commerçant, résidant à Yirimadio (Dakaba-carré) notion de voyage et le statut vaccinal. II a été hospitalisé au service des maladies infectieuses du CHU-Point G, salle $\mathrm{x}$, lit $\mathrm{N}^{\circ} \mathrm{y}$ et avec un dossier sans numéro.

Histoire de la maladie :

Le début de la symptomatologie remonterait au 11 Juin 2019, marqué par des céphalées, une myalgie, une douleur dorsale et articulaire irradiant aux deux chevilles dans un contexte fébrile. II n'y avait pas de notion de déplacement du cas. II a consulté un Médecin dans le cabinet $\mathrm{B}$ à Yirimadio le 12/06/2019 où un diagnostic 
initial de crise hypertensive a été posée, avec institution d'un traitement médicamenteux fut instauré en ambulatoire sans succès. II était constitué de Ceftriaxone injectable (non documenté); Vitamine B.Complexe injectable et Novalgin injectable en perfusion dans du Ringer Lactate; Novalgin comprimé, Méthyldopa comprimé et Furosemide comprimé en ambulatoire. Devant la persistance de la fièvre et l'apparition d'une raideur cervicale douloureuse, il a été référé, au service des maladies infectieuses du CHU-Point G, le 18 Juin 2019 où le diagnostic de cas suspect de méningite a été posé. Le prélèvement du Liquide Céphalo-Rachidien (LCR) a été fait et envoyé au laboratoire de I'INRSP. Le résultat préliminaire (test rapide) est revenu positif, le 19 Juin 2019, aux méningocoques W135 et $Y$.

Antécédents : il a été noté que le patient a eu à faire une Méningite en 1993, à Coro à l'âge de 18 ans, avec traitement médical soldé de succès. Cette information n'était pas documentée.

Prise en charge: Le Traitement institué était : Ceftriaxone, Dexaméthasone, Paracétamol injectable, sérums salé et glucosé $5 \%$. L'évolution était favorable de jour en jour, jusqu'à la guérison complète sans séquelle nombre de jour de traitement.

Potentiel de diffusion de la maladie : Dans sa famille, nous avons trouvé qu'une de ses filles nommée BT, âgée 3 ans, était traitée traditionnellement depuis le 17/06/2019 pour fièvre, céphalées et vomissement. Elle n'était pas fébrile et ne présentait aucun autre signe méningé. Nous l'avons mis sous surveillance matin et soir par un ASC et l'évolution a été favorable. L'enfant était complètement vacciné au programme élargi de vaccination (PEV) de routine y compris contre la méningite $A$.

\section{Discussion}

Ce patient était un commerçant et vivait dans un quartier populaire. Ces facteurs augmenteraient son contact étroit avec d'autres populations. Nous savons que la contamination se fait par les voies aériennes et donc, il se retrouverait à risque de cette maladie. II contamination est favorisée par la situation climatique et les mois de mai et juin font partis des 4 mois de pics comme trouvé dans l'étude de Dembélé $L$ et al. (5). Nous pouvons supposer que la contamination se serait passée en mai avec la période d'incubation qui varie généralement entre 2 et 10 jours (1). Cette tendance au Mali est appuyer par d'autres études (6).

II s'agit d'un patient qui récidive de méningite 26 ans après. Ce qui pose des questions quant aux facteurs qui ont concourus à faire la maladie. Nous savons que cette maladie n'apporte pas d'immunité. Aussi, le PEV ne vaccine les enfants que contre les souches $A$. cette vaccination a été introduite en 2010 et nous n'avons pas d'information si le patient en avait bénéficié. Les cas de récidive ont été beaucoup décrits dans la littérature et synthétisés dans la revue systématique de Tebruegge $\mathrm{M}$. et Curtis N. en 2008 (7). Beaucoup de définitions de récidive y ont été décrites et notre patient en répond. II s'agit entre autre d'une épisode de méningite qui survient au moins 3 semaines après la guérison de la première (7). Malheureusement, nous ne pouvons pas certifier le premier épisode de notre patient.

La prise en charge n'avait pas été bonne au premier contact avec le cabinet. II aurait eu une hypothèse diagnostic non conforme. D'où un traitement inadéquat qui aurait permis à la maladie d'évoluer. Cela pose le problème de compétence des agents de santé et d'insuffisance de présence des médecins dans les structures qu'ils créé.

La gestion du patient au niveau local n'a bénéficié de la collaboration entre le cabinet et le CSRéf ou CSCom. Ce qui dénote de l'insuffisance de collaboration entre structures publiques et privées, particulièrement dans le domaine de la surveillance épidémiologique.

La prise en charge a été rapide et adéquate au niveau du $\mathrm{CHU}$. Le prélèvement du LCR, qui est de moins en moins pratiqué dans les structures périphériques, a été effectué et le résultat préliminaire a été rapidement obtenu de I'INRSP. Cette rapidité entre dans l'amélioration de la prise en charge surtout à ce niveau ultime de la référence dans notre pyramide sanitaire.

Limites de l'étude :

L'absence d'échange en mode face à face des Responsables du service d'infectiologie du CHU-Point G et du cabinet, associé à l'absence d'information précises sur l'épisode précédente de méningite ont limité notre étude en terme d'information permettant de mieux comprendre les circonstances de survenu du présent épisode. Nous n'avons pu faire l'investigation conformément aux directives de l'OMS (8) A ces limites, s'ajoute la contrainte liée à l'insuffisance de moyens logistiques pour les différents déplacements.

\section{Conclusion}

II s'agissait d'un patient de 42 ans avec méningite W135 et $Y$ ayant bénéficié d'une meilleure prise en charge au $\mathrm{CHU}$ contrairement au cabinet privé. La guérison a intervenu sans séquelles. II n'y a pas eu d'autres dans l'aire de santé avec la surveillance de la famille par l'ASC. II serait intéressant de mener une étude plus approfondie sur les facteurs favorisant la méningite chez cette personne et renforcer la collaboration entre les structures publiques et privées dans la commune en matière de surveillance épidémiologique.

\section{Actions de santé publique}

Briefing du personnel du cabinet par rapport à son implication dans la surveillance épidémiologique.

\section{Références}

1. OMS. Guide Technique Méningite [Internet].

OMS, Sous Bureau d'Abéché; [cité 1 nov 2019].

Disponible sur:

http://origin.who.int/hac/crises/tcd/chad_guide_tehnique_ meningite.pdf 
2. La méningite [Internet]. https://www.passeportsante.net/. 2012 [cité 1 nov 2019]. Disponible sur:

https://www.passeportsante.net/fr/Maux/Problemes/Fiche .aspx?doc=meningite_pm

3. Morvan J. Epidémies de méningite en Afrique, Ghana et Burkina Faso [Internet]. MesVaccins.net; 2019 [cité 17 nov 2019]. Disponible sur:

https://www.mesvaccins.net/web/news/13459-epidemiesde-meningite-en-afrique-ghana-et-burkina-faso

4. INRSP, DNS, LCV, DNSV. Directive Nationale des prélèments. INRSP, Bamako; 2017.

5. Dembélé L, Konaté I, Goita D, Oumar A, Sangaré S, Coulibaly B, et al. Utilisation des facteurs climatiques pour la surveillance de la fréquence des occurrences de méningite/ paludisme à Bamako. Rev Malienne Infect Microbiol. 2016;2016(8):33-42.

6. Al-Gahtani YM, Bushra HEE, Al-Qarawi SM, AlZubaidi AA, Fontaine RE. Epidemiological investigation of an outbreak of meningococcal meningitis in Makkah (Mecca), Saudi Arabia, 1992. Epidemiol Infect. déc 1995;115(3):399-409.

7. Tebruegge M, Curtis N. Epidemiology, Etiology, Pathogenesis, and Diagnosis of Recurrent Bacterial Meningitis. Clin Microbiol Rev. 1 juill 2008;21(3):519-37. 8. OMS. Investigation d'un cas de Neisseria meningitidis du sérogroupe $A$ dans un pays de la ceinture de la méningite [Internet]. [cité 3 nov 2019]. Disponible sur:

http://www9.who.int/csr/disease/meningococcal/Investiga tion-de-cas-de-NmA-protocole.pdf 